vol. 33 - $n^{\circ} 2$ et $3 \mid 2017$

Dire la violence des frontières. Mises en mots de la migration vers l'Europe

\title{
Migrations de retour dans les Alpes italiennes : mobilités, « cittadinanza » et sentiment d'appartenance
}

Return Migrations in Italian Alps: Mobilities, "Citizenship" and Belonging Migraciones de retorno en los Alpes italianos: movilidades, "ciudadanía» y sentimiento de pertenencia

\section{Blanchard Melissa et Sirna Francesca}

\section{(2) OpenEdition}

\section{Journals}

Édition électronique

URL : https://journals.openedition.org/remi/8634

DOI : 10.4000/remi.8634

ISSN : $1777-5418$

Éditeur

Université de Poitiers

Édition imprimée

Date de publication : 1 septembre 2017

Pagination : 301-322

ISBN : 979-10-90426-59-7

ISSN : 0765-0752

Référence électronique

Blanchard Melissa et Sirna Francesca, « Migrations de retour dans les Alpes italiennes : mobilités, " cittadinanza » et sentiment d'appartenance », Revue européenne des migrations internationales [En ligne], vol. 33 - n² et 3 | 2017, mis en ligne le 01 septembre 2019, consulté le 16 avril 2022. URL : http://journals.openedition.org/remi/8634; DOI : https://doi.org/10.4000/remi.8634 


\section{Note de recherche}

\section{Migrations de retour dans les Alpes italiennes : mobilités, "cittadinanza " et sentiment d'appartenance}

\section{Blanchard Melissa ${ }^{1}$ et Sirna Francesca ${ }^{2}$}

\section{Introduction}

Les migrations de retour, loin d'être un phénomène marginal, ont concerné la majorité des migrants italiens. Des 27 millions d'Italiens émigrés depuis I'unification de la Péninsule (dont la déclaration date de 1861 et l'achèvement de 1871), plus de la moitié sont rentrés en Italie (Sanfilippo, 2001). Parmi les 8 millions de rapatriés enregistrés entre le début du XXe siècle et 1976, $30 \%$ sont revenus entre 1965 et 1976 (Corti, 2005 : 128). La migration de retour est un phénomène qui a concerné de nombreuses familles dans plusieurs nations, mais l'interprétation de ce phénomène n'est pas toujours aisée à cause de la rareté des données (Ravenstein, 1885 ; Devoto, 1993 et 2003 ; Sanfilippo, 2005) ${ }^{3}$. Les analyses des migrations n'étudient pas forcément les mouvements de retour. Lorsqu'elles le font, le retour devient l'objet d'études qui ne prennent pas en considération le pays où les migrants ont séjourné parfois pendant de nombreuses années ${ }^{4}$. Le " retour " est un aspect très important des flux migratoires non seulement d'un point de vue quantitatif, mais également pour les effets qu'il a pu avoir sur le pays d'origine ${ }^{5}$.

\footnotetext{
1 Anthropologue, Chercheure associée IDEMEC/URMIS, Maison Méditerranéenne des Sciences de I'Homme, 5 rue du Château de I'Horloge, BP 647, 13094 Aix-en-Provence cedex 2 ; mblanchard@mmsh.univ-aix.fr

2 Sociologue, Chargée de recherche CNRS, Centre Norbert Elias, EHESS/Aix-Marseille Université, Centre de la Vieille Charité, 2 rue de la Charité, 13002 Marseille;

francesca.sirna@univ-amu.fr

3 Ravenstein (1885: 168) avait déjà pointé la rareté des données et le manque d'attention pour les migrations de retour comme facteur " défaillant " des analyses sur les mouvements de populations dès la fin du XIXe siècle.

$470 \%$ des migrants rentrant en Italie dans la deuxième moitié du XXe siècle venaient de pays européens. Sur les $30 \%$ restant, $15 \%$ provenaient des États-Unis et $33 \%$ d'Amérique du Sud (source : Centro Studi Emigrazione, 1978). Sanfilippo (2005) a souligné la difficulté d'interpréter les migrations de retour dont l'étude est complexe et doit prendre en considération les évolutions dans les deux pays (d'immigration et d'émigration). Devoto (1993 et 2003) a également suggéré la nécessité d'interpréter les migrations comme phénomène circulaire et multidirectionnel dont le " retour " est une partie constitutive. La nécessité d'insérer le retour dans l'étude des migrations, en tant qu'acte constitutif de l'émigration, a aussi été longuement défendue par Sayad (2006).
}

5 Cerase (1974 et 2001) analyse ce phénomène en tant que facteur de changement économique et social du pays d'origine. 
Dans les Alpes, en particulier, les départs et les retours des émigrants se sont succédé tout au long du XXe siècle jusqu'à nos jours, se superposant aux anciennes pratiques de va-et-vient propres aux migrations de métier, temporaires et à courte distance, qui ont caractérisé cet espace géographique pendant des siècles (Albera et Corti, 2000 ; Audenino, 2009).

Dans cette note de recherche, nous comparerons les parcours de deux groupes d'émigrants et de descendants d'émigrants originaires de deux régions des Alpes italiennes, le Piémont et le Trentin, " rentrant " dans leur région d'origine ou dans celle de leurs ancêtres, entre la crise économique des années 1970 et les années $2010^{6}$. Analyser les parcours de migrants revenant de pays européens proches et de pays d'outre-Atlantique nous aidera à relever les éventuelles différences et similitudes dans les parcours, dans les pratiques et dans le rapport au territoire. Est-ce que la proximité entre le lieu d'origine et le lieu d'immigration détermine des mobilités différentes, voire plus "fluides ", entre ces deux lieux ? Est-ce que cela conduit à des taux de retours plus importants ?

L'intérêt du raisonnement par cas (Livet, 2005) requiert une explication : ici, les parcours individuels sont un exemple qui présente des similarités avec d'autres cas. L'analyse détaillée des parcours permet de pointer les différences entre les deux groupes ainsi que de reconstruire les dynamiques qui façonnent I'action collective. Le cas a également une fonction de "sursomption " montrant comment une règle générale se décline dans une situation singulière ou comment des hypothèses générales se modifient les unes les autres sur le cas considéré.

Peu d'études (Martini et Rygiel, 2009) ont proposé une analyse comparative des flux migratoires européens et d'outre-Atlantique ; cette note de recherche propose également de contribuer à la réflexion sur les effets de la distance/ proximité sur les pratiques et les perceptions que les acteurs ont de leur trajectoire. II veut aussi apporter une contribution aux études sur les migrations alpines en questionnant la complexité des pratiques et des espaces dans lesquels ces acteurs se déploient (Viazzo, 2000 ; Fontaine, 2005). II s'agira ainsi d'analyser deux " systèmes migratoires ". Par système migratoire, nous faisons référence à la définition de Kritz et Zlotnik (1992) pour qui ce concept désigne un groupe de pays ou de régions qui échangent des quantités relativement élevées de migrants I'un avec l'autre. L'apport de l'analyse systémique (Sayad, 1977 ; Fawcett, 1989) relève de la prise en compte simultanée de plusieurs dimensions : celle de l'émigré/immigré ; celle des liens historiques, culturels, coloniaux, technologiques qui se nouent entre un lieu (d'émigration) et un autre (d'immigration); celle des contextes économiques, démographiques, sociaux et politiques dans lesquels se déploient ces flux. Cette approche permet aussi de mettre en évidence la pluralité des réseaux qui lient les acteurs, les interactions entre les

6 Ces migrants, hommes et femmes, ont été rencontrés dans le cadre de deux recherches distinctes. L'une issue d'une thèse en sociologie soutenue à I'EHESS en décembre 2007, sous la direction de Paul-André Rosental, et l'autre dans le cadre d'une recherche postdoctorale en sociologie à l'université de Trento, sous la direction de Giuseppe Sciortino. Les méthodes $\mathrm{d}^{\prime}$ enquête qualitative employées sont celles de I'entretien approfondi et de l'observation participante. L'étude du contexte socio-économique a couplé le travail d'enquête de terrain. Les échanges avec les acteurs locaux (maires, employés municipaux/régionaux, responsables d'associations) ont nourri nos observations et analyses. 
différents types de flux (de départs et de retours), ainsi que les politiques migratoires tant au niveau national qu'international.

D'abord, nous soulignerons l'importance des flux de retour au sein des migrations italiennes, en nous attardant sur la spécificité du revenir dans les sociétés alpines. Deuxièmement, nous poursuivrons avec l'analyse critique de la catégorie " migration de retour " afin de mieux révéler la polysémie qui lui est propre et de mettre en évidence sa valeur heuristique. Ensuite, nous retracerons les parcours migratoires des deux groupes de migrants pris en considération. Nous soulignerons en particulier deux points. D'une part, la place du retour au sein des parcours de mobilité individuels et familiaux afin de saisir les effets de ces derniers sur les pratiques des acteurs. D'autre part, nous questionnerons le rapport entre ces retours et le sentiment d'appartenance nationale/régionale/ villageoise, en analysant le sens que le retour prend selon les générations, le cycle de vie et les contextes historiques d'origine et d'émigration.

\section{Migrations et modèle alpin de mobilité}

L'émigration de masse a caractérisé I'histoire du royaume d'Italie dès son unification (1861). Au cours du XXe siècle, l'émigration à destination des pays transocéaniques, mais aussi européens, semble être un élément structurel au développement de l'Italie afin d'équilibrer la fragilité de la structure industrielle et le retard du système productif agricole (Sanfilippo, 2001). Après un ralentissement au cours des deux conflits mondiaux, l'exode vers l'étranger reprend avec une importance considérable en $1946^{7}$ et se poursuit tout au long des années 1950 et 1960 jusqu'à la première moitié des années $1970^{8}$. Les départs concernent toutes les régions de la Péninsule, mais leur nombre est particulièrement élevé dans les îles (en particulier la Sicile), le Sud, ainsi que les zones montueuses de I'arc alpin (Giarrizzo, 1987). À partir de 1975 et à la suite de la crise pétrolière internationale, les rapatriements dépassent les départs (123000 retours contre 93000 départs) (Cerase, 2001). Dans les années 1980, les départs et les retours stagnent et s'équilibrent. Les années 1990 ne modifient pas cette tendance ${ }^{9}$, qui se poursuit jusqu'en 2008, avec pourtant des chiffres dix fois moins importants (47 000 départs par an contre 43000 retours). La décennie 1990-2000 suit donc l'orientation des décennies précédentes : diminution des flux, à la fois de départs et de retours, équilibre entre émigration et rapatriements (40 000 personnes environ) et division géographique des flux en sortie (vers l'Europe) et en entrée (ces derniers en provenance des pays de l'Amérique latine) (Sanfilippo, 2003a). Depuis la crise économique de 2008, le nombre des retours diminue légèrement, de 37326 en 2005 à 29271 en 2014, tandis que les départs augmentent de manière significative (41 991 en 2005 à 88859 en 2014)

\footnotetext{
7225000 départs ; 293000 dans les années 1950, 264000 au cours des années 1960 (Cerase, 2001).

81961 fut l'année de nombreuses expatriations (387 000) ; celles-ci diminuèrent légèrement en 1962 (229 000 départs). Entre 1970 et 1975, les départs vers et les retours depuis l'étranger s'équilibrent : 132000 départs, contre 129000 retours (Cerase, 2001).

9 Entre 1990 et 1999, on décompte 468223 départs vers l'étranger et 426473 inscriptions à I'anagrafe, le registre de l'état civil, pour raisons de rapatriement.
} 
montrant une réactivation de l'émigration italienne ${ }^{10}$.

Ceci étant, le décompte des " retours " des émigrants s'avère problématique pour plusieurs raisons. D'une part, la difficulté de saisir ce phénomène à travers les catégories statistiques utilisées d'habitude (King, 1986 ; Rallu, 2007 ; Douki, 2013) ; d'autre part, le caractère temporaire et informel des déplacements rend leur enregistrement difficile ${ }^{11}$. Cette difficulté d'obtenir des données officielles à l'échelle régionale a été également constatée lors de nos recherches de terrain. Pour en évaluer l'importance, nous avons fait référence aux rapports sur les bénéficiaires des politiques régionales d'aide au retour, couplés à nos observations. Nos estimations semblent indiquer qu'un habitant sur dix est aujourd'hui issu d'une migration de retour au Trentin ${ }^{12}$ et un sur cent au Piémont ${ }^{13}$.

L'émigration italienne, loin d'être un phénomène monolithique, se caractérise par l'existence de différentes modalités du migrer (Gabaccia, 2000). Notre analyse, fondée sur l'approche régionale (Sanfilippo 2002), entend éclairer ces spécificités. Une morphologie migratoire discontinue traduit l'existence de dynamiques et de stratégies familiales non uniformes, dont il n'est possible de rendre compte qu'à travers la reconstruction des trajectoires des migrants. La comparaison entre deux régions de l'arc alpin, le Piémont, situé à l'extrémité occidentale, et le Trentin, situé sur le versant oriental, illustrera l'influence des deux systèmes migratoires (Reyneri, 1979 ; Bonifazi et Heins, 1996 ; Sanfilippo, 2001) sur les modalités du retour.

Les Alpes ont été, depuis l'Ancien Régime, au centre de flux de mobilité humaine. Le modèle alpin de mobilité ${ }^{14}$, mis à jour par les études d'historiens et d'anthropologues à partir des années 1980 (Viazzo, 1989 ; Albera et al., 1998), a invalidé la vision braudelienne, longtemps célébrée, de " la montagne fabrique d'hommes à l'usage d'autrui " (Braudel, 1966 : 29). Ces analyses ont ainsi révélé que la montagne, loin d'être un milieu socialement et économiquement pauvre et à forte pression démographique, a été un lieu où la nuptialité tardive, un haut pourcentage d'alphabétisation et un système de production mixte (agropastorizia) ont calibré l'accès aux ressources. L'émigration saisonnière des habitants des vallées alpines a été une stratégie supplémentaire d'ajustement aux contraintes écologiques (Viazzo, 1989). Aussi, l'émigration prend la forme d'une mobilité temporaire de métier. Ceux qui partent sont des entrepreneurs, des colporteurs, des artisans qualifiés, des maîtres se déplaçant en hiver vers les régions limitrophes ou plus éloignées où leurs services sont demandés, pour revenir à la saison estivale (Audenino, 1990 ; Fontaine, 1993 ; Corti, 1995).

10 Élaboration à partir des données de l'Istituto Nazionale di Statistica (ISTAT) (Migrantes Fondazione, $2016: 10)$.

11 L'anagrafe, bureau qui enregistre les changements de résidence des ménages (à l'intérieur et à l'extérieur du territoire national italien), n'a pas le pouvoir de contrôler lesdits changements. Les individus ou les familles qui quittaient un lieu de résidence, devaient eux-mêmes en informer le bureau de I'anagagrafe (Douki, 2013).

12 http://www.mondotrentino.net/banche_dati/

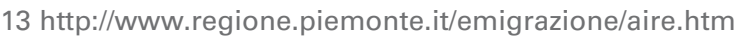

14 Nous faisons ici référence à la littérature sur les migrations alpines et les zones montueuses du pourtour méditerranéen (Fontaine, 2005). Le terme " modèle " renvoie à I'analyse des migrations à l'échelle d'un territoire circonscrit, la vallée, la région, etc., que ces études ont très bien illustré. 
Cette longue pratique de la mobilité, typique des régions montagneuses (Albera et Corti, 2000 ; Fontaine, 2005), confluera ensuite dans la grande émigration italienne dès 1870 jusqu'au milieu des années 1970 (Audenino, 2009). Les nombreuses études citées montrent ainsi une continuité entre la mobilité saisonnière " de métier " à courte distance et les flux d'émigration de masse intercontinentaux et transocéaniques. Nos deux terrains montreront également I'inscription des pratiques migratoires dans la longue histoire familiale confirmant ce que Hägerstand (1957) avait déjà appelé " l'effet d'entraînement " : les migrations présentes étant liées aux migrations passées. L'analyse des deux groupes régionaux ici retenus révèlera ainsi la prégnance des contextes d'origine et d'émigration sur le rapport au territoire et à la nationalité, ainsi que sur les pratiques spatiales (Simon, 2006).

\section{Pour une appréhension critique du " retour " des migrants}

La littérature sur le retour des migrants recouvre une multiplicité de phénomènes, allant des retours temporaires et cycliques, aux retours conçus comme définitifs, jusqu'aux visites sporadiques à la famille. Plusieurs auteurs ont proposé des tentatives de catégorisation des retours sur la base de critères divers, tels que la durée du retour ou de la migration, la modalité de la mobilité (circulaire, unilinéaire, pendulaire), l'intention du migrant, l'issue du projet migratoire, etc. (Bovenkerk, 1974 ; Gmelch, 1980 ; King, 1986).

Ici, nous entendons souligner la difficulté de définir le revenir au sein des phénomènes de mobilité. En effet, des retours conçus comme définitifs peuvent toujours être suivis d'un nouveau départ, au gré des nécessités et des opportunités. Ensuite, nous pointons la complexité du rapport entre mobilité internationale et mobilité interne (King et Skeldon, 2010). En effet, il n'est pas aisé de définir comme " retour " la mobilité de migrants ou des descendants d'émigrants s'installant dans une localité autre que celle d'où ils sont originaires (Michalon, 2007 ; Zúñiga et Hamannb, 2015). Enfin, nous voudrions mettre en évidence que la notion de retour rend compte souvent de deux processus différents. Elle est, en effet, employée pour les émigrants ainsi que pour leurs descendants nés à l'étranger et n'ayant jamais vécu dans le pays vers lequel ils " reviennent ". Les parcours ici retracés s'inscrivent dans cette pluralité de situations.

En prenant en considération les "retours " des émigrants et des descendants d'émigrants dans les deux régions alpines du Piémont et du Trentin, nous ne nous intéressons pas exclusivement au retour des retraités, qui ont été le thème central des principales études sur le retour (Catani, 1986 ; Petit, 2007 ; King et Christou, 2010), mais aux " retours " qui concernent plusieurs générations. Très peu de littérature analyse la réinstallation des descendants d'émigrants dans le pays de leurs ancêtres et les recherches qui le font sont assez récentes (Tsuda, 2004 ; Christou, 2006 ; Potter et Phillips, 2006 ; Sardinha, 2011). Ce phénomène est appelé " migration des racines " (Wessendorf, 2007), " mobilité contre-diasporique " (Olsson et King, 2008) ou " migration de retour ethnique " (Brubaker, 1998 ; Tsuda, 2009). II doit être distingué des voyages sporadiques que les descendants d'émigrants entreprennent pour rendre visite à la famille ou au village de leurs parents ou grands-parents. Ce dernier type de mobilité 
a engendré une floraison d'études, qui mettent en lumière la complexité des représentations identitaires qui se révèle lors de ces voyages (Baldassar, 2001 ; Sirna, 2009), ainsi que l'ambigüité de la démarche, se situant à mi-chemin entre le tourisme (Sampaio et al., 2014) et le pèlerinage des racines (Sanchini, 2010). Le retour des descendants d'émigrants est défini par deux lieux, le pays d'origine et le pays de destination, qui sont l'opposé spéculaire de ceux caractérisant la migration de leurs parents ou ancêtres (Christou et King, 2010). Le pays d'origine des descendants d'émigrants, en effet, est le pays de destination de leurs parents, tandis que leur pays de destination est le pays d'origine de leurs parents. Les implications de ce "renversement " sont fondamentales pour comprendre le lien entre nationalité et retour.

La notion de retour implique, enfin, de porter notre regard sur les pratiques transnationales des émigrants européens, considérées par de nombreuses études comme une caractéristique de l'époque contemporaine (Basch et al., 1994 ; Portes, 2001). Pourtant, l'émigration italienne fut toujours empreinte de pratiques familiales transnationales, de circulations et d'échanges de biens, de personnes et d'idées (Foner, 2001 ; Gabaccia et lacovetta, 2002). L'analyse des parcours migratoires de deux groupes de migrants des Alpes italiennes nous aidera à étayer ces propos.

\section{Les différents types de " retour "}

\section{Le cas des émigrants et des descendants d'émigrants trentins}

Comme pour le reste de la Péninsule, à partir des années 1970, le Trentin a enregistré un déclin de l'émigration qui, dans les décennies précédentes, avait causé le dépeuplement de vallées entières et une augmentation des retours des émigrants et de leurs descendants. Ce mouvement se poursuit jusqu'à aujourd'hui, mais, comme déjà évoqué plus haut, il est difficile d'en calculer l'extension réelle.

En prenant en compte les pays d'installation des émigrants qui reviennent, on relève le poids de ce contexte sur l'issue plus ou moins favorable de l'émigration (Spagnoli, 2009). Selon le témoignage du coordinateur des mesures pour le rapatriement définitif de la province de Trente, les émigrants trentins et leurs descendants rentrent beaucoup moins fréquemment du Canada, de l'Australie et des États-Unis ou de France, tandis qu'ils sont nombreux à revenir des pays d'Amérique du Sud. Si les premiers pays offrent aux émigrants et à leurs descendants de bonnes conditions d'insertion socio-économique, qui les ont encouragés à rester, les pays d'Amérique latine, souvent soumis à des périodes de crise économique et politique profonde, sont les lieux de nouveaux départs ${ }^{15}$. Les bouleversements sociaux et économiques liés à l'installation des dictatures

$15 C^{\prime}$ est le cas du retour des colons du Chili à l'époque de la réforme agraire du gouvernement Allende d'abord et de la dictature de Pinochet ensuite; et du retour des émigrants d'Argentine au cours de la dictature militaire et suite à la crise économique désastreuse provoquée par le gouvernement Menem. Concernant les destinations européennes, une vague relativement importante a été enregistrée en provenance de la Bosnie à l'époque de la guerre 1992-1995. Dans ce cas, c'étaient surtout des jeunes hommes, descendants d'Italiens, qui " revenaient " au Trentin pour éviter le recrutement militaire. Ces retours restent cependant numériquement contenus (Groselli, 2000). 
au Chili et en Argentine au cours des années 1970, notamment, ont poussé un nombre important d'émigrants et de descendants d'émigrants à quitter leurs pays d'implantation pour s'embarquer dans une " migration à rebours ". Pour les émigrants partis dans la deuxième après-guerre, ces départs coïncident aussi avec la fin d'un cycle de vie.

Entre 2010 et 2013, nous avons réalisé une enquête ethnographique auprès de vingt familles revenant d'Argentine et du Chili au Trentin. Au cours de cette recherche, nous avons rencontré plusieurs générations de migrants et de descendants d'émigrants. Si la majorité de ceux qui " revenaient " étaient des émigrants ou des enfants d'émigrants, ayant quitté le Trentin enfants ou étant nés en Amérique latine, nous avons pu aussi rencontrer des descendants d'un lointain ancêtre trentin. Nous avons ainsi décelé trois grands types de "retour " selon lesquels ce dernier se réalise et selon le sens qui lui est attribué dans le cadre d'un plus ample projet de vie familial ou individuel.

\section{Le retour nostalgique}

Le premier type de retour est celui des familles, parties dans la première moitié des années 1900 et revenues au cours des années 1970-1980. Elles reviennent soit à la suite de l'échec du projet migratoire (comme pour les émigrants partis au cours des années $\left.1950^{16}\right)$, soit parce qu'elles considèrent que ce dernier a atteint son but. Dans ce cas, les familles reviennent à l'âge de la retraite du père ou à un moment où celui-ci estime avoir accumulé suffisamment d'économies pour pouvoir rentrer. Les retours ouvrent alors sur un rebondissement vers de nouvelles opportunités professionnelles : principalement des entreprises familiales dans le secteur de I'hôtellerie.

Mario est issu d'une famille de scieurs de la haute Val di Sole. Son arrièregrand-père et son grand-père se déplaçaient déjà, au cours de I'hiver, vers les plaines de Vérone et de Mantoue pour travailler comme artisans dans l'industrie du bois. Aujourd'hui retraité, Mario raconte son expérience d'émigrant au Chili :

" J'ai voulu rentrer en 1970 parce que j'avais de la nostalgie, je voulais rentrer auprès des miens, revoir ma mère et mes frères. J'avais trente-sept ans. Je suis venu au Chili dans les années 1950, quand l'État italien envoyait les familles en Amérique du Sud. J'y étais allé avec mes parents et toute ma famille, dix frères. Mais mon père n'a pas résisté, parce qu'à nous, on nous avait promis un terrain cultivable, au Nord. À la place, on nous a donné des terres que même pas une foreuse y aurait fait un trou. Impossibles à cultiver.

\footnotetext{
16 Les émigrations à destination de l'Amérique latine étaient souvent encadrées par des politiques de recrutement promues par les Etats récepteurs, visant à coloniser des terres soustraites aux populations autochtones, ainsi qu'à créer une nouvelle classe prolétaire urbaine d'ascendance européenne (Groselli, 2000). La dernière grande vague de départs collectifs enregistrée au Trentin se dirigea vers le Chili dans les années 1950 au cours de deux expéditions. Le départ de colons était organisé directement par la région Trentin-Haut-Adige en collaboration avec le gouvernement chilien et à l'initiative des États-Unis qui le finançaient avec les fonds du Plan Marshall. Le contrat signé par les colons prévoyait qu'à leur arrivée une maison et des terres leur soient assignées à un prix très avantageux. La seconde expédition, cependant, eut une issue désastreuse, les colons découvrant que les terrains qu'ils avaient achetés étaient arides et impossibles à cultiver. Beaucoup d'entre eux, qui avaient vendu leurs maisons et leurs terres au Trentin, se retrouvèrent à la limite de la survie et furent rapatriés par les autorités consulaires au cours des années 1970 et 1980 (Groselli, 2011).
} 
Alors, nous avons abandonné et avons cherché quelque chose, mais on ne parlait pas l'espagnol, là ils sont pauvres aujourd'hui encore, et à l'époque ils l'étaient davantage.

Mon père, désespéré, a rebroussé chemin, il est rentré au village. Un an après, il a rappelé toute la famille. Mais moi, je me suis dit "pauvreté là-bas, pauvreté ici, à ce point je reste ici". À l'époque j'avais dix-huit ans. Je suis resté seul, mais j'avais des amis, des Italiens, on avait fait des petites colonies où on se retrouvait, ou bien on se connaissait dans un boulot ou dans l'autre."

Avec les économies rapportées du Chili, Mario a ouvert un premier, puis un deuxième hôtel dans la vallée d'où il était originaire, devenue une destination touristique prisée pour les sports d'hiver.

Les pratiques spatiales desTrentins de retour révèlent que ceux-ci s'installent majoritairement dans les mêmes lieux d'origine. Ces localités de montagne, qui ont un caractère rural, ont connu depuis les années 1960, un essor économique lié aux pratiques touristiques. Il s'agit de lieux qui offrent des opportunités intéressantes pour les émigrants de retour qui ont aussi contribué au développement de ces mêmes opportunités. Ainsi, il n'y a pas de circulation entre les lieux d'origine et les anciens lieux d'émigration (Chili, Argentine).

\section{Le " retour " comme nouvelle émigration}

Le deuxième type de retour est celui des enfants ou de descendants d'émigrants qui sont nés et ont grandi au Chili ou en Argentine et dont les parents restent dans le pays d'émigration. Ces Chiliens ou Argentins d'origine trentine, " reviennent " dans le cadre de ce qu'on peut considérer un nouveau projet migratoire. Venant de pays traversant des crises économiques et politiques à répétition, ils espèrent trouver dans le pays d'origine de leurs parents ou de leurs ancêtres le même Eldorado que ces derniers étaient partis chercher sur I'autre rive de l'océan. Ils partent souvent dans le cadre d'une migration familiale de jeune couple au seuil de la vie active, mariés soit avec un autre descendant $d^{\prime}$ Italiens, soit avec un Chilien/Argentin, et cherchant en Italie des opportunités pour démarrer une nouvelle vie professionnelle et fonder une famille. Antonella retrace son parcours :

" Je suis Argentine, mais citoyenne italienne née à l'étranger. Mon père est né en Argentine d'une mère trentine et ma mère est émigrée de la Vénétie quand elle avait quinze ans, après la Deuxième Guerre mondiale. Ma mère me racontait les souffrances des émigrants jusqu'à apprendre la langue, et pour moi ça a été la même chose. Tu arrives et tu ne sais pas parler, même si tu as beaucoup de diplômes tu sais que tu dois faire des travaux humbles. Moi, je me suis retrouvée comme ça, à quarante ans. C'était très difficile psychologiquement, parce que j'aime faire le ménage chez moi, mais je n'aime pas le faire comme métier. J'ai dû le faire et j'ai dû apprendre, car ce n'est pas la même chose que de faire le ménage chez toi ou dans un hôtel. La première période, arrivée ici avec quatre enfants mineurs, j'ai dû travailler. Mon mari, lui aussi avec un master, a dû faire le maçon, chose qu'il n'avait jamais faite. Moi j'étais éducatrice en Argentine, je me suis mise en jeu complètement pour mon pays, pour les plus démunis. Ensuite, je me suis fatiguée, j'ai perdu l'espoir en l'Argentine... Après la crise de 2002, quand nous avons eu cinq présidents en une semaine, nous avons décidé de venir. Mes enfants commençaient le lycée et je me suis dit... et après, ici ? En Argentine, tout le monde étudie, car il n'y a pas d'emploi. Et puis nous avons tous des diplômes universi- 
taires et on va faire... ou tu pars en Europe quand tu as vingt-cinq ans. Et je me suis dit "eux, ils vont partir en Europe et moi, je reste ici ?" Ce jour-là, j'ai eu un déclic. Ma mère, mon père, ils venaient tous les ans et me disaient qu'au Trentin il y avait des facilités, tu peux essayer. Nous, nous avons bénéficié de tout, avec beaucoup de bureaucratie, mais nous avons bénéficié d'aides et de facilités. Je ne suis pas arrivée avec la valise sans savoir où dormir. On nous donne un logement, on nous offre un premier travail, un contrat de six mois pour la collectivité."

Ces " nouveaux migrants de retour " se rendent dans leur pays natal, en Amérique latine, très rarement, pour rendre visite à leurs parents : les coûts du déplacement ne permettent pas une véritable circulation. Cependant, ceci n'est pas un facteur de distension des liens affectifs et familiaux, qui sont maintenus via les nouvelles technologies de communication (Skype, Internet).

\section{Le retour " refuge "}

Le troisième type de retour est celui d'enfants ou de petits-enfants d'émigrants, nés en Amérique latine, qui se sont dirigés vers l'Italie pour fuir la persécution politique suite aux dictatures instaurées au Chili ${ }^{17}$ et en Argentine ${ }^{18}$. Des enfants d'émigrants, engagés dans les luttes juvéniles et estudiantines, trouvent alors refuge dans la terre de leurs ancêtres. Celle-ci, cependant, est pour eux principalement un lieu d'exil. En effet, revenir au pays d'où sont partis leurs parents ou leurs grands-parents correspond à un choix contraint, imposé par les circonstances aussi souvent que par leur famille afin de les éloigner d'un engagement politique dangereux. Le contexte premier d'investissement affectif, politique et existentiel de ces enfants d'émigrés reste leur pays de naissance, le Chili et l'Argentine, et même après quelques décennies de permanence en Italie, la douleur engendrée par ce déracinement forcé est grande. Cependant, beaucoup d'entre eux restent auTrentin, parce que les enfants sont nés ou scolarisés sur place, ils ont une situation économique stable et ne veulent pas tout remettre en cause pour un " retour " dans un pays où leur situation serait incertaine.

Rosa est née en Argentine d'un père trentin, qui était parti pour l'Amérique du Sud après avoir tenté une première émigration en Belgique avec son père où ils avaient travaillé dans les mines de charbon. Rosa raconte son arrivée en Italie:

" Je suis Argentine, de Buenos Aires. Je suis venue en 1982, à cause de l'histoire, parce que de 1976 à 1983 nous avons eu une dictature. Au cours de la dernière période de la dictature, je fréquentais l'université et donc, pour des causes de force majeure, en l'espace d'une semaine je me suis retrouvée chez mes grands-parents au Trentin.

La situation était très délicate et ce n'est pas le cas d'en parler. On laisse ce qu'on était... J'ai mis de côté mon rêve, parce que j'étudiais l'histoire avec une spécialisation en archéologie, j'ai dû tout révolutionner. Je suis restée dans le domaine du tourisme parce que quand même ça me plait. Quand je suis arrivée, je ne croyais pas rester. Mon père, qui est d'ici, m'a mise dans un avion et m'a envoyée au Trentin en l'espace d'une semaine. J'avais laissé, en plus de mes études, ma vie là-bas, mes sentiments et tout. Et ceci je l'ai trainé pendant deux ans. Après deux ans, je me suis rendu compte que je ne rentrerai plus jamais. Ça a été très dur."

17 Dictature du général Augusto Pinochet, 1973-1990.

18 Dictature militaire de la " Révolution argentine », 1966-1973 ; juntes militaires, 1976-1983. 
Ces exilés ne se rendent que très rarement dans leur pays de naissance, non seulement à cause de la distance et du coût du voyage, mais aussi, en raison de la souffrance engendrée lors de ces retours sur les lieux du passé.

\section{Les retours nostalgiques des Piémontais : rebondir, échouer ou réussir?}

La reconstruction des parcours des Piémontais ${ }^{19}$, hommes et femmes, revenus de leur séjour à l'étranger, montre aussi que ce déplacement peut avoir un rôle différent selon le moment du cycle de vie et la durée de la migration. Les départs s'inscrivent, ici aussi, dans des histoires familiales marquées par la mobilité : tous les Piémontais rencontrés avaient des membres de leur famille ayant déjà séjourné à l'étranger. Les enfants de ces Piémontais ont souvent été rencontrés en France : ils ne suivent que rarement leurs parents dans ces retours, pour deux raisons principales. D'une part, le système des prestations sociales françaises, étant plus généreux qu'en Italie, les encourage à rester en France même lorsqu'ils font face à l'instabilité professionnelle. D'autre part, la proximité du pays d'origine des parents leur permet d'effectuer des visites régulières. Giovanni et Filippo, les deux fils d'Elio (dont le parcours sera développé plus bas) décident de rester en France lorsque leur père repart en Italie. Ils travaillent tous les deux comme maçons et Giovanni explique ainsi son choix :

"Ici, je pouvais trouver un travail plus facilement. Puis, j'avais ma copine et on voulait avoir des enfants et on avait droit à l'appartement, les aides et tout le reste... Au Piémont, j'avais mes parents, mais bon, pas d'aides pour la famille, pas la santé [sécurité sociale], pas l'école gratuite, pas de [allocation] chômage...

Le Piémont, c'est bon pour les vacances... Je suis né ici [en France]. "

L'analyse des parcours migratoires de vingt-cinq couples de Piémontais, ayant émigré dans le Sud-Est de la France après la Deuxième Guerre mondiale, nous a permis d'identifier, à l'intérieur de la catégorie des " retours nostalgiques ", des perceptions différentes que les acteurs ont de leurs trajectoires.

\section{Quand revenir permet de " rebondir "}

Lorsque le retour depuis l'étranger a lieu pendant l'âge actif, il est rarement définitif. Il s'agit de retourner dans le pays d'origine, mais non dans le lieu de résidence d'avant l'émigration. Pour ces migrants, le retour n'est pas une installation définitive dans le village d'origine, mais le début d'un périple régional qui les voit évoluer dans le monde ouvrier des années 1960. Retourner signifie se rapprocher de sa famille, sans forcément emménager dans le lieu d'origine. Souvent, la permanence à l'étranger n'a pas été très longue et les migrants, face aux difficultés de stabiliser leur condition professionnelle, préfèrent repartir dans le pays d'origine, mais là où ils trouveront des opportunités. II faut analyser ce type de retour en lien avec la pluralité des contextes que les migrants appréhendent dans leur périple. À titre d'exemple, certains des Piémontais rencontrés avaient déjà émigré au Brésil avant d'arriver en France et, ensuite, revenir au Piémont. L'instabilité politique que traverse le pays d'immigration - par exemple

19 L'analyse des parcours des Piémontais révèle que les retours ne peuvent s'inscrire qu'à l'intérieur de la catégorie "retours nostalgiques " décrite dans le paragraphe précédent. 
le Brésil au milieu des années 1950 - la crise économique et/ou une dictature (qui débutent en 1964 au Brésil) peuvent être à I'origine d'un développement économique "chaotique " du pays et de la faillite de nombreuses entreprises (Sanfilippo, 2003b). Le retour, dans ce contexte, ne peut pas être considéré comme un " échec " d'insertion et d'adaptation. Les migrants essaient plutôt d'obtenir des conditions professionnelles plus confortables et plus "sûres ". Trois éléments concourent au retour. D'une part, le choix de la période d'émigration vers le pays d'immigration qui peut être peu favorable à l'installation. D'autre part, la " courte durée " du séjour qui ne permet pas d'atteindre la stabilité professionnelle.

L'oncle d'un des migrants rencontrés, arrivé au Brésil pendant la guerre, ne reviendra en Italie qu'au début des années 1970 avec une "petite fortune ", comme le dit son neveu. Lorsque la crise économique se produit, il est quasiment en " fin de carrière " et, surtout, a développé des liens sur place que son neveu n'a pas et qui lui permettent de "résister " aux difficultés.

Le troisième élément déterminant est le développement économique et industriel de I'Italie du Nord, où habite une partie de la famille des migrants. Ainsi, revenir n'est qu'une étape dans un parcours qui est réajusté au fur et à mesure que le migrant accède, ailleurs, à d'autres ressources nécessaires à la poursuite de son projet. Il s'agit d'une inflexion du projet. Revenir signifie suivre les opportunités les plus accessibles, rebondir vers d'autres espaces professionnels.

\section{Retour d'échec}

Le deuxième type de retour concerne des migrants ayant subi les conséquences de la crise pétrolière et qui étaient plus âgés que ceux du premier groupe : le " rebondissement " n'a pas eu la même signification.

Le parcours d'Elio en est un exemple. Ancien paysan/maçon, il émigre en France pendant dix-huit ans, comme avaient fait son père et son grand-père avant lui. II travaille dans une société de maçonnerie. Au début des années 1970, son patron vend son entreprise et licencie la moitié des ouvriers, dont Elio qui se retrouve sans emploi à quarante-huit ans. Après deux mois de recherche, il décide de rentrer en Italie avec sa femme et I'un de ses trois enfants. II réussit à être embauché dans l'industrie chimique (de peintures) comme ouvrier grâce à son cousin. Le salaire n'est pas très élevé et sa femme est obligée de rechercher un travail. Aujourd'hui à la retraite, le couple vit dans un logement HLM de la périphérie de Turin. Deux fils vivent en France avec leurs familles et leur fille cadette vit et travaille àTurin comme comptable dans une entreprise alimentaire. La femme d'Elio regrette d'avoir quitté la France et de s'être éloignée de ses enfants.

Le retour de nombreuses familles, bien qu'elles aient réussi à se réinsérer économiquement et socialement, traduit une déception, un échec, mais pas au sens d'incapacité à s'insérer dans le lieu d'immigration. Ces migrants craignent de subir les conséquences à long terme de la crise économique à un âge avancé. Ainsi la présence d'un réseau familial dans une autre ville du Piémont, souvent à Turin, ville industrielle et chef-lieu de la région, détermine leur choix. Ainsi Elio : 
" J'ai attendu deux mois, j'ai demandé [du travail] à tous ceux que je connaissais.

Mais ce n'était pas le bon moment. Tout le monde craignait pour son propre poste... À Turin, j'avais deux frères et des cousins. J'ai appelé et ils m'ont dit qu'y avait la possibilité de travailler. Je suis parti. Ma femme voulait pas, mais comment faire ?! On est rentré avec la petite. Mes fils travaillaient, ils avaient leurs copines. Ils sont restés. Vous savez, parfois on n'a pas trop le choix. Il faut s'en sortir. J'ai fait ce que j'ai pu... "

Si le retour représente l'opportunité de se soustraire au chômage dans une période de crise, plus que l'accomplissement du projet, il marque également des nouvelles pratiques spatiales. En effet, revenir en Italie matérialise la séparation avec une partie de la descendance, car les enfants ne suivent pas leurs parents dans cette migration (contrairement aux Trentins), probablement du fait de la proximité des deux lieux d'émigration et d'immigration. La gamme des choix possibles pour ces ouvriers qui ne sont plus jeunes (au moment de leur licenciement) est restreinte et elle détermine un parcours descendant et un éloignement des affects. Ainsi, souvent ces migrants se rendent plusieurs fois par an chez les enfants installés en France : I'ancien espace migratoire devient le lieu où passer les vacances.

\section{Retour comme accomplissement du projet}

In fine, le retour peut être l'accomplissement du projet jamais abandonné. Le projet migratoire prévoit un retour qui peut être différé, ajourné, latent, mais toujours présent dans les récits des migrants. La réalisation du dessein initial peut prendre plusieurs années et correspondre souvent à la fin de la vie active. Dès lors, le retour comme installation définitive fait place à un usage de l'espace sillonné par un " pendularisme " (Miranda, 1996). Ces pratiques caractérisent aussi bien les " migrants de retour " que les migrants propriétaires d'un logement dans le lieu d'origine et dont la résidence administrative reste dans le lieu d'immigration. Cette modalité rappelle le phénomène que Baldassar (2001) appelle "visits home ". L'exemple suivant permet de mieux comprendre cette conception du retour.

En 1938, les parents de Maria (âgée de deux ans) émigrent à Marseille. Son père gère un petit magasin d'alimentation où sa mère travaille aussi. Ils ont un deuxième enfant qui naît en France. Chaque été, la famille revient dans le village où Maria rencontre Francesco, son futur mari. Les fiancés se marient, Francesco rejoint Maria à Marseille et travaille dans le magasin de son beaupère. Au moment de la retraite, le père de Maria décide de revenir dans le village d'origine. Pour ce faire, il entreprend les travaux de rénovation de la maison parentale et s'y installe avec sa femme. Maria, Francesco, le mari et leurs deux enfants passent les vacances dans le village chez les grands-parents. Au moment de la retraite, Maria et Francesco décident de revenir dans le village : leurs enfants sont partis habiter dans d'autres régions de France et les parents de Maria sont toujours vivants et très âgés. Contrairement à d'autres femmes rencontrées, Maria n'a jamais souhaité rester en France, car le retour dans le village ne représentait pas un éloignement de ses enfants :

"De toute façon, ils étaient déjà partis [les enfants]. Un à Paris, l'autre à Nantes.

On serait resté seul à Marseille, alors que mes parents étaient dans le village et avaient plus besoin de moi que mes enfants. On est revenu, mais on part trois, quatre fois par an, 
pendant les vacances scolaires pour garder les petits [petits-enfants]. En été, ils viennent nous voir, passer quelques semaines à la montagne. Pour les vacances d'hiver aussi.

Comme ça, on se voit souvent. Mes petits-enfants nous voient plus que les autres grandsparents qui vivent en France ! Alors, la distance, ce n'est pas un problème ! "

II ne s'agit pas d'une obligation, mais d'un choix. Ce retour pourrait être dû aux meilleures conditions économiques espérées dans le pays d'origine. Ceci est un facteur influant sur le choix de retour. Cependant, d'autres facteurs président à ce choix : la présence des parents âgés dans l'espace d'origine ; la mobilité des enfants qui ne justifie plus la présence des migrants dans l'espace d'immigration ; la possibilité, en revenant, de les accueillir dans un lieu touristique (renommé pour sa station de ski) et, enfin, la capacité de se rendre en France très régulièrement. Leur retour devient une "large circulation" (Rallu, 1998) entre le Piémont et la France. Pour d'autres anciens migrants venus à l'âge de la retraite dans le village d'origine ou dans des centres urbains de basse montagne, "l'installation " devient aussi une "pluralité de mouvements " (Gmelch, 1980), surtout lorsque les enfants ou d'autres membres de la famille résident à l'étranger ou dans les villes de la plaine ${ }^{20}$.

Le " pendularisme " - ou la circulation - ne marque pas un relâchement du rapport avec les origines, mais une réorganisation de l'espace qui a une valeur symbolique importante pour toute la famille (Corti, 1994 ; Rosental, 1999) ${ }^{21}$.

\section{Retour et nationalité : les différents usages selon la génération et la période historique}

Les retours, décrits plus haut, révèlent l'existence d'une pluralité de rapports à l'espace et à la nationalité22. Dans le cas particulier de l'Italie, la présence d'un fort régionalisme (campanilismo), l'existence de traditions, de langues locales et de situations socio-économiques différentes laissent peu de place à un sentiment d'appartenance nationale (Levi, 1979). Les migrations italiennes ont été le résultat de départs de villages, de régions, de villes avant même d'être une manifestation reconductible à la nation dont I'unification remonte à 1861 . Ce n'est pas accidentel si le mot italien pour exprimer la nationalité est cittadinanza, qui manifeste, à l'origine, le lien entre l'individu et la ville (città) et non avec la nation. Ainsi le rapport à la cittadinanza serait influencé par les différents contextes nationaux, historiques, socio-économiques, géographiques qui sont traversés par les migrants, mais également par leur position dans le cycle de vie

\footnotetext{
20 De nombreuses études sur les migrations de retour à l'âge de la retraite ont mis en évidence ce phénomène d'installation dans un autre lieu que celui d'origine; et il s'agit souvent de centres urbains de taille moyenne que les anciens migrants trouvent plus proches du style de vie qu'ils ont connu dans le pays d'immigration (Gmelch, 1980 ; Miranda, 1996 ; Audenino, 1990 ; Pellegrino et Santos, 1989 ; Meloni, 1997 ; Rallu, 1998).

21 Cette mobilité des migrants retraités peut être déterminée également par une conception de l'espace et de la distance différente ou modifiée par l'expérience migratoire : les migrants élaborent un " territoire " à l'intérieur duquel ils se déplacent de manière "fluide ", sans que cela soit un événement traumatisant.
}

22 Entendue ici comme l'appartenance à une nation déterminée, au sens du lien juridique et politique qui rattache un individu à un Etat. 
et dans la généalogie migratoire ${ }^{23}$.

Au cours des entretiens des deux groupes de migrants, lorsqu'il a été question de définir leur appartenance à un espace national (d'origine ou d'immigration), tous les émigrants rencontrés ont évoqué une affiliation régionale, puis locale (la vallée et ensuite le village). En revanche, les enfants et les descendants des émigrants se sont souvent définis comme des Chiliens, des Argentins ou des Français. Le rapport à la nationalité italienne semble se négocier en fonction des circonstances. Les stratégies individuelles et familiales jouent ainsi un rôle important dans le " bon " usage de la nationalité. Ces stratégies s'inscrivent à l'intérieur de trois grandes phases historiques : la récession économique des années 1970 ; les crises politiques des pays d'Amérique latine au cours des années 1970-1980; le processus d'attraction de I'Italie à partir des années 1990.

\section{Crise économique des années 1970 et aides au retour}

Les migrants piémontais (ayant gardé la nationalité italienne) installés en France et revenus en Italie à cause et pendant la crise pétrolière des années 1970 ont pu bénéficier des aides étatiques prévues à cet égard.

À ce propos, l'État français prévoyait une prime de 10000 francs pour chaque migrant décidant de rentrer dans son pays d'origine. Cette mesure, initialement prévue pour inciter les rapatriements des populations maghrébines, n'a pas produit le résultat souhaité : des 100000 retours d'immigrés, entre 1975 et 1986, plus de la moitié étaient des Italiens et des Espagnols et seulement un quart des Nord-africains (Tapinos, 1988 ; Schor, 1998). L'administration régionale piémontaise aussi, à partir des années 1980, a mis en place des aides économiques visant le repeuplement des zones rurales, incitant la création d'entreprises et I'achat d'un logement et prévoyant la couverture des frais de déménagement pour toute la famille ${ }^{24}$. Ainsi, nombre de Piémontais revenus au cours de cette période, ont fait valoir leur droit aux deux financements, revendiquant leur nationalité italienne. En revanche, ces aides n'étaient pas accessibles à leurs enfants, de nationalité française, car le critère de la citoyenneté faisait défaut : la reconnaissance de la double nationalité remonte à 1995.

Parmi les émigrants trentins qui reviennent avec leur famille dans le cadre d'un projet de réinstallation familiale, l'origine régionale est souvent employée comme une identité différentielle dans le pays d'émigration, par le biais d'activités collectives avec des co-régionaux. Les émigrants se définissentTrentins, plus qu'Italiens, au Chili et en Argentine. Le recours au soutien économique des politiques d'aide au retour est un avantage auquel certains considèrent $d$ 'avoir droit en vertu de leur origine. Les politiques d'aide au retour de la province deTrente, en vigueur depuis 1980, octroient des fonds pour le rapatriement des émigrants, des descendants d'émigrants et de leurs biens. Elles prévoient aussi de leur fournir un logement et un subside pour faciliter leur réinsertion dans le tissu local ${ }^{25}$.

23 Nous faisons référence à la différence entre l'émigrant et ses descendants, qui reviennent dans le pays des aïeux.

24http://www.piemonteimmigrazione.it/site/index.php?option=com content\&view=article \&id=79\%3Anormativa-regionale-emigrati-di-ritorno\&catid=34\%3Aistituzionale\&ltemid=72\# 25 http://www.mondotrentino.net/rimpatrio_definitivo/ 
Les politiques d'aide au retour de la province de Trente et de la région Piémont constituent un volet d'un ensemble de mesures nationales de repeuplement visant à contrecarrer l'exode rural dans les régions de montagne. Elles font écho à la loi italienne au regard de la transmission de la nationalité, qui postule que le descendant d'un ancêtre italien peut acquérir la nationalité italienne sans limites de génération, pourvu que cet ancêtre soit émigré après $1871^{26}$. Ainsi, une logique d'affinité ethnique insuffle les politiques régionales d'aide au retour, qui considèrent les enfants et les descendants d'émigrants, comme membres d'une nation qui s'étend au-delà des frontières de l'État italien (Joppke, 2005). Ceci leur confère le droit de recouvrer immédiatement la nationalité de la terre de leurs ancêtres et de prétendre à une aide au "retour " (Tsuda, 2010). Il subsiste, cependant, une différence de taille entre les mesures d'aide au retour piémontaises et trentines. Tandis que les premières, dès le début de leur entrée en fonction, ne s'adressent qu'aux émigrants et aux enfants d'émigrants, les secondes intéressaient, jusqu'en 2007, toute personne pouvant prouver leur ascendance trentine, si lointaine soit-elle. Ce n'est qu'après avoir remarqué que ces mesures encourageaient le "retour " d'un nombre trop élevé de " migrants " par rapport à ceux que la province pouvait financer, que des critères généalogiques plus contraignants ont été introduits. À I'heure actuelle, seulement les enfants ou les petits-enfants d'émigrants peuvent prétendre, avec les émigrants, à l'aide au retour au Trentin.

\section{Crises politiques des années 1970-1980 en Amérique latine}

Pour les enfants et descendants d'émigrés trentins qui se rendent au Trentin pour fuir la persécution politique, la double nationalité est un avantage qui se révèle vital. Cependant, leur citoyenneté de cœur, leur pays d'investissement et d'identification, reste au-delà de I'océan : en Argentine ou au Chili.

Les enfants d'émigrés qui sont nés et/ou ont grandi au Chili et en Argentine et encore plus les descendants d'émigrants, même après des années de vie au Trentin, continuent de se sentir et de se déclarer Argentins ou Chiliens. Le pays qui a été le cadre de leur socialisation compte davantage, dans la définition de leur identité personnelle et affective, que le lieu d'origine de leurs parents. Le sentiment d'appartenance n'est donc pas fonction du temps, ni de la nationalité au sens administratif : il dépend plutôt de l'investissement affectif et social des migrants dans les différents lieux de leur existence.

\section{Inversion de la polarité migratoire et vieillissement de la population migrante}

Les enfants et descendants d'émigrés trentins revenus au cours des années 1990 et 2000 ont eu recours presque systématiquement aux politiques d'aide au retour. Dans le cas de ceux dont l'ascendance trentine remontait à une lointaine cinquième génération, il s'agissait d'accomplir une nouvelle émigration dans des conditions économiques favorables. L'ascendance trentine est ainsi redécouverte et réactivée dans une perspective utilitariste. Les individus appartenant à ces différentes générations de migrants peuvent avoir plusieurs nationalités. C'est la condition à laquelle l'on fait référence par le terme transnational

26 http://www.esteri.it/mae/it/italiani_nel_mondo/serviziconsolari/cittadinanza.html 
citizenship (Fox, 2005). D'un point de vue individuel la transnational citizenship peut être considérée comme une "identité en veille ", un élément influençant les choix d'appartenance (Bauböck, 2010). Ainsi, le soutien économique et social que la province de Trente offre, à " ses " émigrés, peut encourager les Chiliens et les Argentins d'ascendance trentine à redécouvrir et à prouver leurs origines, afin de " rentrer " en Italie (Blanchard, 2012).

Pour les Piémontais revenus au cours des années 1990 et 2000 et qui ont souvent acquis la nationalité française, il a été possible de redemander la nationalité italienne et de l'obtenir. Ainsi faisant, même lorsque leurs conditions économiques étaient aisées, ils ont pu prétendre aux aides régionales qui leur ont permis de restructurer ou acquérir un bien immobilier ou entreprendre une activité indépendante.

Le récit des migrants piémontais au sujet du "sentiment d'appartenance " est, lui aussi, assez ambigu : comme pour les Trentins, ils s'identifient plus à la région, voire à la vallée et au village, qu'à la nation. Ainsi, la justification du recours aux aides, évoquée par les migrants, est assez surprenante : il s'agirait de la reconnaissance de leur sacrifice et de leur parcours par l'État italien. Ainsi un migrant piémontais, revenu en 1975 :

"Si en Italie y a eu le boom économique, c'est grâce à nous [les migrants]! Nous avons libéré la place pour que les autres travaillent et nous avons envoyé de l'argent pour les aider. Alors, les aides, c'est le minimum que l'Italie pouvait faire pour nous !"

\section{Conclusions}

L'approche comparative ici proposée a permis de révéler des similitudes et des différences dans les pratiques et dans les perceptions du retour : les Trentins et les Piémontais rencontrés reviennent dans les régions d'origine et souvent avec leurs familles. Les deux groupes font un usage utilitariste de la nationalité et s'identifient davantage au village et à la vallée d'origine qu'à la nation. Cependant, les pratiques spatiales restent différentes. À cause de la distance entre les pays d'immigration et le pays d'origine, le retour des Trentins s'inscrit fréquemment dans une perspective de sédentarité. II comporte une dimension familiale, souvent intergénérationnelle, un projet de réinstallation " à long terme " et un recours plus important aux aides provinciales. En revanche, dans une situation de proximité entre les deux lieux, comme pour les Piémontais, le retour devient une forme de circulation pour plusieurs générations. La proximité permet d'insérer l'espace d'origine des parents dans un territoire où les frontières administratives ne sont pas des obstacles insurmontables (Corti, 1995 ; Rosental, 1999). Ceci expliquerait un moindre nombre de dossiers de demandes d'aide au retour des Piémontais. Cette proximité est aussi à l'origine de " pratiques administratives d'invisibilité " : tous les Piémontais ne déclaraient pas leur départ en France.

La comparaison entre les migrations de retour des Alpins en provenance de I'Europe ou des pays d'outre-Atlantique montre que I'affiliation nationale pour les migrants italiens et pour leurs descendants ne joue pas de manière univoque et linéaire dans la genèse du choix et des modalités du retour. Plusieurs facteurs influencent la décision de revenir : la période historique ; les politiques liées à 
I'acquisition et au maintien de la nationalité ; les mesures d'aide au retour et la présence ou absence $d$ 'aides sociales dans le pays d'immigration. Un système de prestations sociales avantageux, comme en France, peut déterminer la décision de ne pas revenir pour les enfants des émigrants. En revanche, l'absence d'aides sociales dans des pays comme le Chili et l'Argentine et la possibilité d'accéder aux financements prévus lors des retours peuvent encourager les émigrants et leurs descendants à " revenir " au Trentin.

Pour conclure, nous pouvons nous interroger pour savoir s'il y a une spécificité de la mobilité alpine concernant le retour. Nous pensons que celle-ci ne relève pas tellement des modalités du retour ou de ses motivations qui, au contraire, caractérisent les mouvements de contre-mobilité en général. La spécificité alpine réside plutôt dans les histoires familiales concernées depuis l'Ancien Régime par ces déplacements, et dans la capacité à recourir à la mobilité comme stratégie de déploiement familiale et intergénérationnelle : l'espace est une ressource à utiliser de manière pragmatique afin de poursuivre un projet familial et individuel (Grandi, 1998).

\section{Références bibliographiques}

Albera Dionigi et Corti Paola (2000) Migrations montagnardes dans l'espace méditerranéen. Esquisse d'une analyse comparative, Mélanges de l'école française de Rome. Italie et Méditerranée, 112 (1), pp. 359-384.

Albera Dionigi, Dossetti Manuela e Ottonelli Sergio (1998) Società ed emigrazioni nell'alta Valle Varaita in età moderna, Bollettino storico-bibliografico subalpino, 86 (1), pp. 117-169.

Audenino Patrizia (2009) Quale ritorno? Tempi, significati e forme del ritorno nelle Alpi italiane dall'Otto al Novecento, Histoire des Alpes-Storia delle AlpiGeschichte der Alpen, 14, pp. 57-73.

Audenino Patrizia (1990) Un mestiere per partire. Tradizione migratoria, lavoro e comunità in una vallata alpina, Milan, Franco Angeli, 281 p.

Baldassar Loretta (2001) Visits home: migration experiences between Italy and Australia, Carlton South, Melbourne University Press, 396 p.

Basch Linda, Glick-Schiller Nina and Szanton Blanc Cristina (1994) Nations Unbound:Transnational Projects, Postcolonial Predicaments and Deterritorialized Nation-States, New York, Gordon and Breach, 356 p.

Bauböck Rainer (2010) Cold constellations and hot identities: Political theory questions about transnationalism and diaspora, in Rainer Bauböck and Thomas Faist Eds., Diaspora and transnationalism, Amsterdam, Amsterdam Universtity Press, pp. 295-321.

Blanchard Melissa (2012) Donne straniere e lavoro autonomo in Trentino. Un confronto tra i percorsi socio-economici delle immigrate et delle emigranti di ritorno, Centro Scenari Migratori e Mutamento Sociale, Rapport de recherche, Université de Trento, $79 \mathrm{p}$.

Bonifazi Corrado e Heins Frank (1996) Le migrazioni di ritorno nel sistema migratorio italiano: un riesame, Studi emigrazione-Etudes Migrations, 30 (122), pp. 273-303. 
Bovenkerk Frank (1974) The Sociology of Return Migration: A Bibliographic Essay, The Hague, Martinus Nijhoff, $76 \mathrm{p}$.

Braudel Fernand (1966) La Méditerranée et le monde méditerranéen à l'époque de Philippe II, Paris, Armand Colin, 400 p.

Brubaker Rogers (1998) Migrations of Ethnic Unmixing in the "New Europe", International Migration Review, 32 (4), pp. 1047-1065.

Catani Maurizio (1986) Les migrants et leurs descendants entre devenir individuel et allégeance chthonienne, Cahiers Internationaux de Sociologie, 81, pp. 281-298.

Cerase Francesco Paolo (2001) L'onda di ritorno: i rimpatri, in Piero Bevilacqua, Andreina De Clementi e Emilio Franzina Eds., Storia dell'emigrazione italiana. Partenze, Roma, Donzelli, pp. 113-126.

Cerase Francesco Paolo (1974) Expectations and reality: a case study of return migration from the United States to Southern Italy, International Migration Review, 8 (2), pp. 245-262.

Christou Anastasia (2006) Narratives of Place, Culture and Identity: SecondGeneration Greek-Americans Return "Home", Amsterdam, Amsterdam University Press, $264 \mathrm{p}$.

Christou Anastasia and King Russell (2010) Imagining "Home": Diasporic Landscapes of the Greek-German Second Generation, Geoforum, 41 (4), pp. 638-646.

Corti Paola (2005) Storia delle migrazioni internazionali, Bari, Laterza, 146 p.

Corti Paola (1995) L'émigration italienne : historiographie, anthropologie et recherche comparatiste, Revue Européenne des Migrations Internationales, $11(3)$, pp. 5-18.

Corti Paola (1994) Paesi d'emigranti. Mestieri, itinerari, identità collettive, Milan, Franco Angeli, 296 p.

Devoto Fernando (2003) Italiani in Argentina: ieri e oggi, Altreitalie, 27, pp. 4-17.

Devoto Fernando (1993) Emigrazione italiana: un fenomeno di lunga durata, Altreitalie, 10, pp. 1-8.

Douki Caroline (2013) Compter les " retours " d'émigrants dans I'Italie du début du XXe siècle : conventions statistiques, libéralisme économique et politique publique, Revue Européenne de Migrations Internationales, 29 (3), pp. 11-32.

Fawcett JamesT. (1989) Networks, Linkages, and Migration Systems, International Migration Review, 23 (3), pp. 671-680.

Foner Nancy (2001) Transnationalism then and now: New York immigrants today and at the turn of the twentieth century, in Héctor R. Cordero-Guzmán, Robert C. Smith and Ramón Grosfoguel Eds., Migration, Transnationalization, and Race in a Changing New York, Philadelphia, Temple University Press, pp. 35-57.

Fontaine Laurence (2005) Montagnes et migrations de travail. Un essai de comparaison globale (XVe-XXe siècles), Revue d'histoire moderne et contemporaine, 52 (2), pp. 26-48.

Fontaine Laurence (1993) Histoire du colportage en Europe, XVe-XIXe siècle, Paris, Albin Michel, 336 p. 
Fox Jonathan (2005) Unpacking transnational citizenship, Annual Review of Political Science, 8, pp. 171-208.

Gabaccia Donna R. (2000) Italy's many Diaspora, Seattle, University ofWashington Press, 264 p.

Gabaccia Donna R. and lacovetta Franca (2002) Introduction, in Donna R. Gabaccia and Franca lacovetta Eds., Women, gender and transnational lives. Italian workers of the world, Toronto, Toronto University Press, pp. 3-41.

Giarrizzo Giuseppe (1987) Sicilia oggi (1950-86), in Maurice Aymard e Giuseppe Giarrizzo Eds., La Sicilia. Storia d'Italia. Le regioni dall'Unità a oggi, Turin, Einaudi, pp. 603-696.

Gmelch George (1980) Return Migration, Annual Review of Anthropology, 9, pp. 135-159.

Grandi Casimira (1998) Emigrazione alpina al femminile: lo spazio del possibile (sec. 17-20), Histoire des Alpes-Storia delle Alpi-Geschichte der Alpen, 3, pp. $49-62$.

Groselli Renzo Maria (2011) Un urlo da San Ramon: la colonizzazione trentina in Cile, 1949-1974, Trento, Fondazione Museo Storico Trentino, 647 p.

Groselli Renzo Maria (2000) Storie della emigrazione trentina, Trento, L'Adige, $408 \mathrm{p}$.

Hägerstand Torsten (1957) Migration and area. Survey of a sample of swedish migration fields and hypothetical considerations on their genesis, Migration in Sweden, 13, pp. 27-158.

Joppke Christian (2005) Selecting by Origin: Ethnic Migration in the Liberal State, Cambridge, Harvard University Press, 344 p.

King Russell (1986) Return migration and regional economic problems: an overview, in Russell King Ed., Return Migration and Regional Economic Problems, London, Croom Helm, pp. 1-37.

King Russell and Christou Anastasia (2010) Diaspora, migration and transnationalism: insights form the study of second generation "returnees", in Rainer Bauböck and Thomas Faist Eds., Diaspora and transnationalism, Amsterdam, Amsterdam University Press, pp. 167-183.

King Russell and Skeldon Ronald (2010) "Mind the Gap!" Integrating Approaches to Internal and International Migration, Journal of Ethnic and Migration Studies, 36 (10), pp. 1619-1646.

Kritz Mary and Zlotnik Hania (Eds.) (1992) International Migration System: A global approach, Oxford, Clarendon Press, 368 p.

Levi Giovanni (1979) Regioni e cultura delle classi popolari, Quaderni Storici, $15(2)$, pp. 720-773.

Livet Pierre (2005) Les diverses formes de raisonnement par cas, in Jacques Revel et Jean-Claude Passeron Éds., Penser par cas, Paris, EHESS, pp. 229-254.

Martini Manuela et Rygiel Philippe (2009) Genre et travail migrant. Mondes atlantiques, XIXe-XXe siècles, Paris, Publibook, 198 p.

Meloni Benedetto (Ed.) (1997) Famiglia meridionale senza familismo. Strategie economique, reti di relazione e parentela, Rome, Meridiana, 208 p. 
Michalon Bénédicte (2007) Les retours en migration : une notion polysémique, des formes migratoires multiples, in Véronique Petit Éd., Migrations internationales de retour et pays d'origine, Paris, CEPED, pp. 27-45.

Migrantes Fondazione (2016) Rapporto Italiani nel Mondo 2016. Sintesi, Roma, Tav Editrice, $43 \mathrm{p}$.

Miranda Adelina (1996) Migrants et non-migrants d'une communauté italienne, Paris, L'Harmattan, 153 p.

Olsson Erik and King Russell (2008) Introduction: Diasporic return, Diaspora: $A$ Journal of Transnational Studies, 17 (3), pp. 255-261.

Pellegrino Pierre et Santos Paulo (1989) Espace : identité et altérité des mobiles. La perspective du retour au Portugal, Espaces et Sociétés, 54-55, pp. 142-155.

Petit Véronique (Éd.) (2007) Migrations internationales de retour et pays d'origine, Paris, CEPED, 208 p.

Portes Alejandro (2001) Introduction: the debates and significance of immigrant transnationalism, Global Networks, 1 (3), pp. 181-193.

Potter Robert and Phillips Joan (2006) Both Black and Symbolically White: The "Bajan-Brit" Return Migrant as Post-colonial Hybrid, Ethnic and Racial Studies, 29 (5), pp. 901-927.

Rallu Jean-Louis (2007) L'étude des migrations de retour: données de recensement, d'enquête et de fichiers, in Véronique Petit Éd., Migrations internationales de retour et pays d'origine, Paris, CEPED, pp. 47-56.

Rallu Jean-Louis (1998) Étude des migrations de retour : données de recensement, d'enquête et de fichiers, Démographie, analyse et synthèse, Actes du Séminaire de San Miniato, 3 (17-19 décembre), La Sapienza, Rome, INED/UCL, pp. 89-98.

Ravenstein Ernst Georg (1885) The Laws of Migration, Journal of the Statistical Society of London, 48 (2), pp. 167-235.

Reyneri Emilio (1979) La catena migratoria. Il mercato del lavoro nelle zone di esodo e di arrivo, Bologna, II Mulino, $355 \mathrm{p}$.

Rosental Paul-André (1999) Les sentiers invisibles. Espaces, familles et migrations dans la France du XIXe siècle, Paris, Éditions de l'EHESS, 255 p.

Sampaio Sofia, Simoni Valerio and Isnart Cyril (2014) Tourism and transformation: negotiating metaphors, experiencing change, Journal of Tourism and Cultural Change, 12 (2), pp. 93-101.

Sanchini Laura (2010) Visiting La Madre Patria: Heritage Pilgrimage Among Montreal Italians, Ethnologies, 32 (2), pp. 235-253.

Sanfilippo Matteo (2005) Problemi di storiografia dell'emigrazione italiana, Viterbo, Sette Città, 266 p.

Sanfilippo Matteo (Ed.) (2003a) Emigrazione e storia d'Italia, Cosenza, Pellegrini Editore, $309 \mathrm{p}$.

Sanfilippo Matteo (2003b) Gli Italiani in Brasile, Viterbo, Sette Città, 46 p.

Sanfilippo Matteo (2002) Problemi di storiografia dellemigrazione italiana, Viterbo, Sette città, $389 \mathrm{p}$. 
Sanfilippo Matteo (2001)Tipologie dell'emigrazione di massa, in Piero Bevilacqua, Emilio Franzina e Andreina De Clementi Eds., Storia dell'emigrazione italiana. Partenze, Roma, Donzelli, pp. 77-94.

Sardinha João (2011) "Returned" Second-Generation Portuguese-Canadians and Portuguese-French: Return Motivations and Sense of Belonging, Journal of Mediterranean Studies, 20 (2), pp. 231-254.

Sayad Abdelmalek (2006) L'immigration ou les paradoxes de l'altérité. Tome 1. L'illusion du provisoire, Paris, Raisons d'agir Éditions, 216 p.

Sayad Abdelmalek (1977) Les trois " âges " de l'émigration algérienne en France, Actes de la Recherche en Sciences Sociales, 15, pp. 59-79.

Schor Ralph (1998) Histoire de I'immigration en France de la fin du XIXème siècle à nos jours, Paris, Armand Colin, 348 p.

Simon Gildas (2006) Migrations, la spatialisation du regard, Revue Européenne des Migrations Internationales, 22 (2), pp. 9-21.

Sirna Francesca (2009) "Revenir au village ". L'expérience du retour au lieu d'origine de Piémontais et de Siciliens émigrés en France, Diasporas. Histoire et sociétés, 14, pp. 33-46.

Spagnoli Frédéric (2009) Migrations de retour vers le Trentin. Vue d'ensemble et étude du cas des "francesi ", Histoire des Alpes-Storia delle Alpi-Geschichte der Alpen, 14, pp. 107-120.

Tapinos Georges (1988) Pour une introduction au débat contemporain, in Yves Lequin Éd., La mosaïque France. Histoire des étrangers et de l'immigration en France, Paris, Larousse, pp. 429-447.

Tsuda Takeyuki (2010) Ethnic return migration and the nation-state: encouraging the diaspora to return "home", Nations and nationalisms, 16 (4), pp. 616-636.

Tsuda Takeyuki (Ed.) (2009) Diasporic Homecomings: Ethnic Return Migration in Comparative Perspective, Stanford, Stanford University Press, 362 p.

Tsuda Takeyuki (2004) When Home Is Not the Homeland:The Case of Japanese Brazilian Ethnicn Return Migration, in Fran Markowitz and Anders Stefansson Eds., Homecomings: Unsettling Paths of Return, Lanham, Lexington Books, pp. 125-145.

Viazzo Pier Paolo (2000) II modello alpino dieci anni dopo, in Dionigi Albera e Paola Corti Eds., La Montagna: una fabbrica di uomini? Mobilità e emigrazioni in una prospettiva comparata (secoli $X V-X X)$, Cavallermaggiore, Gribaudo, pp. 31-46.

Viazzo Pier Paolo (1989) Upland communities, Cambridge, Cambridge University Press, $325 \mathrm{p}$.

Wessendorf Suzanne (2007) "Roots-Migrant": Transnationalism and "Return" among Second-Generation Italians in Switzerland, Journal of Ethnic and Migration Studies, 33 (7), pp. 1083-1102.

Zúñiga Víctor and Hamannb Edmund T. (2015) Going to a home you have never been to: the return migration of Mexican and American-Mexican children, Children's Geographies, 13 (6), pp. 643-655. 


\section{Melissa Blanchard et Francesca Sirna}

\section{Migrations de retour dans les Alpes italiennes: mobilités, "cittadinanza " et sentiment d'appartenance}

Les analyses des migrations n'étudient pas forcément les mouvements de retour. Lorsqu'elles le font, le retour devient l'objet d'études qui ne prennent pas en considération le pays où les migrants ont séjourné, parfois pendant de nombreuses années. À partir d'une lecture critique de la notion de "retour", cette note de recherche compare les parcours de deux groupes d'émigrants et de descendants d'émigrants originaires de deux régions des Alpes italiennes, le Piémont et le Trentin, "rentrant " dans leur région d'origine ou dans celle de leurs ancêtres, entre la crise économique des années 1970 et les années 2010. Peu d'études ont proposé une analyse comparative des flux migratoires européens et d'outre-Atlantique : cette note de recherche propose également de contribuer à la réflexion sur les effets de la distance/proximité sur les pratiques et les perceptions que les acteurs ont de leur trajectoire. II veut aussi apporter une contribution aux études sur les migrations alpines en questionnant la complexité des pratiques et des espaces dans lesquels ces acteurs se déploient.

\section{Return Migrations in Italian Alps: Mobilities, "Citizenship" and Belonging}

Migration studies often neglect return mobility or, on the contrary, the latter becomes the object of studies that do not consider the country where migrants settled, sometimes during years. Building on a critical understanding of "return", this research note compares the paths of two groups of emigrants and emigrants' descendants "coming back" between the economic crisis of the 1970s and the 2010s, in two Alpine regions in Italy (Piedmont andTrentino). Few studies compare European and Atlantic migratory flows: in doing so, this research note analyzes the effects of distance/proximity on actors' practices and perceptions of their trajectories. It also contributes to the studies on Alpine migrations by questioning the complexity of the actors' practices and of the spaces in which they spread.

\section{Migraciones de retorno en los Alpes italianos: movilidades, "ciudadanía" y sentimiento de pertenencia}

Los análisis de las migraciones no estudian forzosamente los movimientos de retorno o, a la inversa, este último es el objeto de estudios que no tienen en cuenta el país dónde los emigrantes permanecieron, a veces, durante numerosos años. A partir de una lectura crítica del "retorno», esta nota de investigación compara las trayectorias de dos grupos de emigrantes y de descendientes de emigrantes originarios de dos regiones de los Alpes italianos, Piamonte y el Trentino, que han "retornado" a su región de origen o a los lugares de origen de sus antepasados entre la crisis económica de los años 1970 y los años 2010. Existen pocos estudios que hayan propuesto un análisis comparativo de los flujos migratorios europeos y Atlánticos: esta nota de investigación propone contribuir a la reflexión sobre los efectos de la distancia/proximidad sobre las prácticas y las percepciones que los actores tienen de su trayectoria. Quiere también aportar una contribución a los estudios sobre las migraciones alpinas interrogando la complejidad de los espacios en los cuales se despliegan, así como las prácticas de los actores. 